\title{
Fragment emission in high-energy heavy-ion reactions
}

\author{
László P. Csernai \\ Institut für Theoretische Physik, Johann Wolfgang Goethe Universität, \\ D-6000 Frankfurt am Main 1, Federal Republic of Germany \\ and Central Research Institute for Physics, H-1525 Budapest, Hungary
}

Horst Stöcker*

Gesellschaft für Schwerionenforschung, D-6100 Darmstadt, Federal Republic of Germany

Pal R. Subramanian, ${ }^{\dagger}$ Gerd Buchwald, Gerhard Graebner, Albrect Rosenhauer, Joachim A. Maruhn, and Walter Greiner

Institut für Theoretische Physik, Johann Wolfgang Goethe Universität, D-6000 Frankfurt am Main 1, Federal Republic of Germany

(Received 9 June 1983)

\begin{abstract}
We present a theoretical description of nuclear collisions which consists of a three-dimensional fluid-dynamical model, a chemical equilibrium breakup calculation for local light fragment (i.e., p, $\mathrm{n}, \mathrm{d}, \mathrm{t},{ }^{3} \mathrm{He}$, and ${ }^{4} \mathrm{He}$ ) production, and a final thermal evaporation of these particles. The light fragment cross sections and some properties of the heavy target residues are calculated for the asymmetric system $\mathrm{Ne}+\mathrm{U}$ at $400 \mathrm{MeV} / \mathrm{N}$. The results of the model calculations are compared with recent experimental data. Several observable signatures of the collective hydrodynamical processes are consistent with the present data. An event-by-event analysis of the flow patterns of the various clusters is proposed which can yield deeper insight into the collision dynamics.
\end{abstract}

\section{$\left[\begin{array}{c}\text { NUCLEAR REACTIONS Relativistic heavy-ion reactions, hydrodynamic } \\ \text { description, nucleon and nuclear cross sections. }\end{array}\right]$}

\section{INTRODUCTION}

Recent experimental results ${ }^{1-5}$ on fragment emission in high energy nuclear collisions can be qualitatively understood as being due to collective flow processes as predicted by the hydrodynamic model. ${ }^{1,5-18}$ In this paper we will present a quantitative comparison of the main experimental results with an extended fluid dynamical model, which also includes a calculation of the light cluster production and their spectra.

The various models constructed so far to describe high energy heavy ion reactions rely on basically different assumptions. In the fireball model, ${ }^{19}$ a global thermal equilibration is assumed among all participant nucleons. In the firestreak model, ${ }^{20}$ this requirement is relaxed to smaller partitions (streaks), while in the hydrodynamic model only local equilibrium is required. On the other hand, the cascade models assume no equilibrium and in their different existing versions different types of equilibration are reached during the collision. The extent of equilibration depends essentially on the mean free path $(\lambda)$ of the nucleons. The mean free path of an impinging proton in the nucleus has recently been determined experimentally to be $\lambda=2.4 \mathrm{fm}^{21}$ However, owing to the increasing temperature and density this value can become much smaller in nucleus-nucleus collisions. First experimental results ${ }^{22}$ yielded $\lambda=1 \mathrm{fm}$. Hence local equilibrium may be achievable and hydrodynamic effects may become important, in particular if heavier systems are investigated. Experimentally the equilibration may be studied by the comparison of the light fragment spectra. ${ }^{23}$

There are a considerable number of theoretical studies of fragment production. Many of these assume that a fireball is produced, which can be characterized by global thermal and chemical equilibrium. They differ from each other in the statistics applied (ideal classical, ${ }^{24-26}$ quantum, ${ }^{20,27-29}$ quantum with interactions ${ }^{30,31}$ ) and in the number of the considered composite fragments, which is usually small, but can go above one hundred by considering all stable and excited states of nuclei up to $A=16^{24}$ In these models the thermal energy is identical to the initial total c.m. kinetic energy. This extreme assumption is mitigated by the consideration of the possible collective flow (or expansion), which may carry a large fraction of the available energy. ${ }^{6,25,32-34}$ In these models, some effects of the expansion or explosion have been studied in simplified spherical geometry, as, e.g., in the blast-wave model $^{35}$ and in the different versions of the hadron chemistry model. ${ }^{36}$ Already in the case of the simple spherical expansion the inclusion of viscous effects into the relativistic hydrodynamic description ${ }^{6}$ can have a strong effect on the observable fragment ratios, as was pointed out recently. ${ }^{33}$ Unfortunately the simple spherical geometry assumed in these models is not very realistic.

In the present calculation we combine the viscous hydrodynamical model (Sec. II) for the collision process with 
a chemical equilibrium breakup model and a final thermal evaporation calculation (Sec. III) to obtain the spectra of the light particles. In Sec. IV we present the results for mean values and fluctuations of the light fragment multiplicities. Section $\mathbf{V}$ contains our results on the double and triple differential cross sections of the light fragments and an analysis of the particle correlations. The formation of heavy fragments and some of their properties are discussed in Sec. VI. An event-by-event analysis for the different fragments is performed in Sec. VII. The conclusions are given in the last section.

\section{THE HYDRODYNAMIC MODEL}

If we derive the hydrodynamic equations from the Boltzmann transport theory, we assume that the system can be characterized by thermally equilibrated local momentum distributions or by distributions close to the equilibrated ones. In the former case we obtain the Euler equations of hydrodynamics, and in the latter the NavierStokes equations, which also include a description of the transport properties of the fluid. If the local momentum distributions are far from the equilibrium ones, a twofluid $^{37}$ or multifluid ${ }^{38}$ description may be applied. In the following discussion we restrict ourselves to laboratory projectile energies up to $400 \mathrm{MeV} /$ nucleon. Since we perform the calculations in the center-of-speed system, the application of nonrelativistic hydrodynamics is sufficient. The classical equations of hydrodynamics can be formulated as conservation equations for mass, momentum, and energy. The local baryon density $n(\vec{r}, t)$ and the flow velocity field $\overrightarrow{\mathrm{v}}(\overrightarrow{\mathrm{r}}, t)$ obey the continuity equation

$$
\partial n / \partial t+\operatorname{div}(n \overrightarrow{\mathrm{v}})=0 .
$$

The conservation of momentum density

$$
\overrightarrow{\mathrm{M}}=\rho \overrightarrow{\mathrm{v}}=n m_{N} \overrightarrow{\mathrm{v}}
$$

is given by

$$
\partial(\overrightarrow{\mathrm{M}}) / \partial t+\operatorname{div}(\overrightarrow{\mathrm{M}} \circ \overrightarrow{\mathrm{v}})=\operatorname{div} P-n \operatorname{grad}(V),
$$

where $\overrightarrow{\mathrm{M}} \circ \overrightarrow{\mathrm{v}}$ denotes the dyadic product and $P$ is the stress tensor given by

$$
\begin{aligned}
P_{i j}= & -p \delta_{i j}+\eta\left[\partial v_{i} / \partial x_{j}+\partial v_{j} / \partial x_{i}-\frac{2}{3} \delta_{i j} \operatorname{div}(\overrightarrow{\mathrm{v}})\right] \\
& +\zeta \delta_{i j} \operatorname{div}(\overrightarrow{\mathrm{v}}) .
\end{aligned}
$$

Here the scalar pressure $p$ is given by the equation of state and the viscous stress tensor involves the shear viscosity $\eta(\rho, T)$ and the bulk viscosity $\zeta(\rho, T)$. The interaction potentials $V$ are not included in the nuclear matter equation of state because of their long-range properties. Accordingly, $V$ is defined as a sum of a Yukawa and a Coulomb contribution, $V_{Y}$ and $V_{C}$. (The way these potentials are determined and the choice of their parameters are described in Ref. 7.) The equation for conservation of energy takes the form

$$
\begin{aligned}
\partial(n E) / \partial t+\operatorname{div}(n E \overrightarrow{\mathrm{v}})= & \operatorname{div}(P \overrightarrow{\mathrm{v}})-\operatorname{div}\left[-\kappa \operatorname{grad}\left(T^{\prime}\right)\right] \\
& -n \overrightarrow{\mathrm{v}} \operatorname{grad}(V),
\end{aligned}
$$

where $\kappa$ is the coefficient of thermal conductivity and $E$ is the energy per baryon (including kinetic and internal energy). In the actual calculations fixed parameters $\eta=10$ $\mathrm{MeV} / \mathrm{fm}^{2} c, \xi=0$, and $\kappa=0$ were used.

To complete the set of equations of motion in the hydrodynamic model, an equation of state has to be specified. This is usually done by giving the binding energy per nucleon at zero entropy as a function of the density, $W_{0}=W_{0}(n)$. For finite entropy per nucleon $s$, the corresponding excitation energy of an ideal Fermi gas is added:

$$
W(n, s)=W_{0}(n)+W_{T}(n, s) .
$$

All other thermodynamic quantities may be obtained easily from $W(n, s)$ :

$$
T=(\partial W / \partial s)_{n}, \quad p=n^{2}(\partial W / \partial n)_{s} .
$$

The binding energy per nucleon at zero entropy, $W_{0}(n)$, is called the compressional energy. One possible form of this function that was employed in the calculations is

$$
W_{0}(n)=K_{0}\left(n-n_{0}\right)^{2} /\left(18 n n_{0}\right)+B_{0},
$$

with $K_{0}$, the incompressibility of nuclear matter, usually set equal to $200 \mathrm{MeV}, B_{0}$ to $-16 \mathrm{MeV}$, and $n_{0}$ to 0.17 $\mathrm{fm}^{-3}$.

Numerical solutions were performed on a grid of cell size $\Delta x=1.2 \mathrm{fm}$. The numerical viscosity which is always present in such calculations increases with increasing cell size. Therefore to reach a good accuracy a very small cell size would be desirable, and then the value of the numerical viscosity could be reduced to a value much smaller than the physical viscosity. However, numerical expenses do not allow us to choose an extremely small cell size, and so the value mentioned above was taken.

From Ref. 13 the numerical viscosity arising from this grid size can be approximated to be approximately the same as the physical value used $\left(10 \mathrm{MeV} / \mathrm{fm}^{2} c\right)$. This is certainly not sufficient for doing systematic studies about viscosity, ${ }^{39}$ but that is not the goal of this paper: Here we are trying to get an overview about the results and the interaction of the hydrodynamical and fragmentation models used. From Ref. 13 it may be expected that variation of $\eta$ (and also of $\Delta x$ leading to different numerical viscosity) should not much alter the qualitative features of the results.

A detailed study of the effects of viscosity on the three-dimensional calculations will be published elsewhere. ${ }^{40}$ The numerical viscosity was found to be 10 (2) $\mathrm{MeV} / \mathrm{fm}^{2} c$ for a cell size of $1.2(0.4) \mathrm{fm}$. The total conservation laws (mass, momentum, and energy) are fulfilled within $0.5 \% .^{40}$ Comparison of temperature and density in the compressed region with values expected from solving the Rankine-Hugoniot equations (which actually require slab geometry) gives agreement to within 5-10\%. The density values obtained numerically are typically below the Rankine-Hugoniot value.

Solving the equations of motion of fluid dynamics in the realistic three-dimensional geometry of a nuclear collision is a very expensive computation. As long as the collision of the two nuclei is supersonic, that is their relative velocity is greater than $0.1-0.2 c$, shock waves will be the 
dominant dissipation mechanism in the hydrodynamic model. Due to the large pressure that builds up in the shocked interaction zone, hydrodynamic models show a preferred sideward emission in central collisions. Note, however, that shock waves are not a necessary prerequisite to getting sidewards emission in hydrodynamics; it can be present in subsonic flows too, when the equation of state is sufficiently stiff. Such predictions, as we will see later, find support from the emission patterns of $\alpha$ particles and protons that have been observbed in high-multiplicityselected events of a particle track detector ${ }^{1}$ and counter data, ${ }^{2-4}$ respectively.

Collisions very close to central do not contribute much to the cross section, so that for measurability considerations it is imperative to examine the behavior at larger, especially intermediate, impact parameters. At intermediate impact parameters a different phenomenon is predicted in the hydrodynamic model, namely the "bounce-off" effect $^{14}$ where the projectile matter as a whole essentially is deflected by the target collectively (Fig. 1).

\section{CHEMICAL EQUILIBRIUM AT BREAKUP: LIGHT FRAGMENT PRODUCTION}

To draw accurate quantitative conclusions regarding the experimental observables in the final state, an evaporation model is attached to the hydrodynamic calculations, $5,6,13,15-17,41,42$ because at late expansion stages of the collision process when the matter is already dilute, the conditions of the hydrodynamic description are not fulfilled. The pressure gradually decreases and a transition from nuclear matter to separate nuclei takes place as, e.g., the surface phase transition in neutron stars. ${ }^{43}$ Here this gradual transition is even more complicated, because we are in a dynamic system, and during the transition the interactions also cease. Small fragments condense out of nuclear matter, forming light nuclei. These then lose contact, owing to the further expansion of the system.

In the model this gradual transition is replaced by a sudden breakup process. The breakup moment is chosen

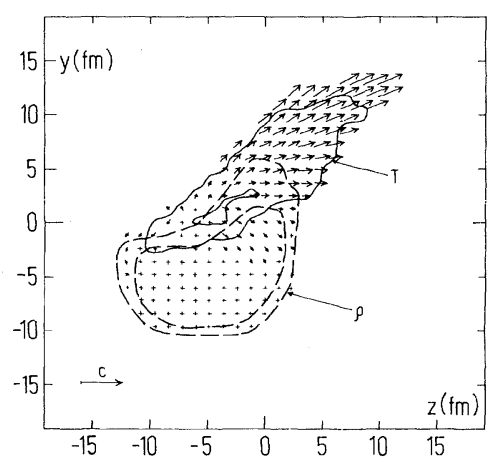

FIG. 1. Density $(\rho)$, temperature $(T)$, and velocity (arrows) distributions in a relativistic heavy ion collision $(\mathrm{Ne}+\mathrm{U} 393$ $\mathrm{MeV} / \mathrm{N})$ in the laboratory system at the breakup moment $(t=35 \mathrm{fm} / c)$. The impact parameter of the collision is $b=6$ $\mathrm{fm}$. The crosses indicate that the flow velocity is $v<0.1 c$. The full contour lines belong to temperatures $T=10$ and $20 \mathrm{MeV}$, the dashed ones to nucleon densities $\rho=0.05$ and $0.1\left(1 / \mathrm{fm}^{3}\right)$. for all impact parameters to be that moment when the maximum nucleon density in the matter is below normal nuclear density. So the average density at breakup is $\rho=0.05-0.07 \mathrm{fm}^{3}$. Unfortunately, the continuous breakup in time, which is used in simple linear and spherical fluid dynamical models ${ }^{6,41,42}$ as well as in a threedimensional calculation ${ }^{17}$ using the particle in cell (PIC) method with a sharp surface, would cause tremendous difficulties in our three-dimensional calculation, especially because of the diffuse nuclear surface and long range $\mathrm{Yu}$ kawa and Coulomb interactions. So we had to choose the sudden breakup assumption as in previous calculations in the hydrodynamic and evaporation model..$^{5,13,15}$ Based on Refs. 36 and 41 we assume that at the breakup moment the chemical and thermodynamical equilibrium is already established locally among the light nuclear fragments. For simplicity we use here simple Boltzmann statistics mainly because of smaller numerical expenses. (The determination of the thermal and chemical equilibrium should be performed separately for each local fluid cell.) Although quantum statistical effects may cause observable differences, ${ }^{27}$ these quantum effects act mainly in the direction of enhanced collective effects. ${ }^{27}$ The relatively limited number of fragments considered and the neglect of quantum effects and decaying states cause, on the other hand, an inaccuracy in $n / p$ and $d / p$ ratios. Thus the densities of different nuclei $\left(i=\mathrm{p}, \mathrm{n}, \mathrm{d}, \mathrm{t},{ }^{3} \mathrm{He},{ }^{4} \mathrm{He}\right)$ are determined by statistical factors ${ }^{24-27}$ :

$$
n_{i}\left(n_{\mathrm{p}}, n_{\mathrm{n}}, T\right)=a_{i} n_{\mathrm{p}}^{Z_{i}} n_{\mathrm{n}}^{N_{i}}
$$

where

$$
a_{i}=\lambda_{T}^{3 A_{i}-3} A_{i}^{3 / 2} 2^{-A_{i}} \exp \left(E_{0}^{(i)} / k T\right)\left(2 S_{i}+1\right)
$$

and

$$
\lambda_{T}=h / \sqrt{2 \pi m_{p} k T}
$$

is the thermal de Broglie wave length. The density of a given fragment $i$ of charge $Z_{i}$ and neutron number $N_{i}$ $\left(A_{i}=N_{i}+Z_{i}\right)$ depends on the common temperature $T$ of the mixture, on the proton and neutron densities $n_{\mathrm{p}}$ and $n_{\mathrm{n}}$, and on the physical properties of the fragment $i$, namely on the ground state energy $E_{0}^{(i)}\left(E_{0}^{(i)}=2.23,8.49\right.$, 7.72, and $28.3 \mathrm{MeV}$ for $i=\mathrm{d}, \mathrm{t},{ }^{3} \mathrm{He}$, and ${ }^{4} \mathrm{He}$, respectively) and spins $S_{i}$. The unknown parameters $n_{p}, n_{n}$, and $T$ can be obtained from the conservation of local baryon number, charge, and energy:

$$
\begin{aligned}
& n=A_{\text {cell }} / V_{\text {cell }}=\Sigma_{i} n_{i} A_{i}, \\
& n(Z / A)=Z_{\text {cell }} / V_{\text {cell }}=\Sigma_{i} n_{i} Z_{i}, \\
& \epsilon+n m_{N}=E_{\text {cell }}^{\mathrm{int}} / V_{\text {cell }}+n m_{N}=\Sigma_{i} n_{i}\left(m_{i}+3 T / 2\right),
\end{aligned}
$$

where $n$ is the baryon density in the fluid cell at the breakup moment and $E_{\text {cell }}^{\text {int }}$ the total internal energy of the fluid cell including binding $\left(k_{\text {Boltz }}=1\right)$. A solution of Eqs. (9) with positive temperature $T$ exists only in those cells where the internal excitation energy is not too low. In the regions where $E^{\text {int }} \ll 8 \mathrm{MeV} /$ nucleon, light fragments cannot be formed [there is no physical solution for Eqs. (9)], but a larger nucleus can be created from the contribu- 
tion of some neighboring fluid cells (See Sec. VI).

It has to be noted that this is not a unique choice of the conserved quantities. In Ref. 17 it is assumed that the entropy is constant during the breakup process. As was pointed out by Scott and Tripathi, ${ }^{44}$ the disassembly of nuclear matter represents a first-order phase transition below a critical temperature of $T_{c} \simeq 18-20 \mathrm{MeV}$, and so an entropy increase may be obtained at the breakup. This shows up as an enhancement of the light fragment emission compared to naive fireball predictions. In our description we do not fix the entropy during breakup and we obtain an increase of the specific entropy of about $10-20 \%$ arising from the fragment formation at low temperatures. At higher temperatures where mainly free nucleons are formed, the entropy hardly changes.

The ratios of the different light nuclear fragments are sensitive to the local temperature at breakup. Since, however, only a minor part of the entropy is produced in the expansion stage ${ }^{6,13,41}$ even in viscous flows, the entropy produced in the compression shock waves can be estimated relatively accurately. The specific entropy $s$ after the breakun moment in each fluid cell can be estimated as

$$
s=\Sigma_{i}\left[\frac{3}{2}+\frac{3}{2} \ln T+q_{i}-\ln n_{i}+\ln g_{i}\right] n_{i} / n,
$$

where

$$
q_{i}=c_{v} \ln \left(2 \pi m_{i} / h^{2}\right)
$$

and $g_{i}$ is the spin degeneracy factor.

In hydrodynamic calculations we can evaluate the local specific entropies before and after the breakup. The latter ones influence the observed light particle cross sections together with the local collective flow. Once the influence of the collective flow in the final state has been extracted, the local thermal excitation of projectile and target can be determined from the ratios of the different light fragments in certain regions of the rapidity space.

In the following calculations a strong average entropy increase occurs at breakup. This increase is for two reasons. The entropy determined after the breakup is the average specific entropy of those regions where light particles $\left(\mathrm{p}-{ }^{4} \mathrm{He}\right)$ are emitted. These are the hottest regions of the collision, where the entropy also is larger. The low entropy of the deeply bound fluid cells does not appear in the entropy of the light particles. On the other hand, the formation of composite fragments leads to temperature and entropy increases in the hot regions also, because we gain the binding energies of the small fragments. These two effects lead to a smaller entropy decrease at low bombarding energies than is expected on the basis of the Rankine-Hugoniot equation with some given equation of state. Other effects ${ }^{31-33}$ also act in this direction so that a quantitative conclusion about the total entropy of the final state as given in Ref. 25 is hardly possible on the basis of the light fragment production ratios alone.

Recent studies of this evaporation process ${ }^{27,30-32}$ show that the consideration of the interactions among the fragments causes strong deviations from the ideal gas assumption, especially for the deuteron to proton ratio. If we increase the density of the deuteron-nucleon gas mixture at a fixed temperature, initially the deuteron density in-

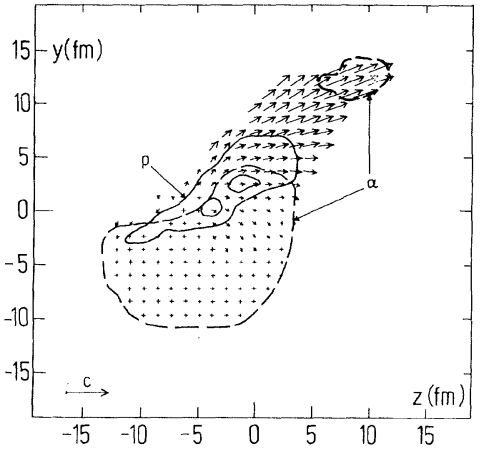

FIG. 2. Proton (p) and alpha particle $(\alpha)$ density contour lines calculated for the breakup configuration of Fig. 1. The protons are formed in the middle hot regions mainly opposite to alphas which are formed at the sides. The contour lines belong to $n_{\alpha}=0.005\left(1 / \mathrm{fm}^{3}\right)$ and $n_{\mathrm{p}}=0.003$ and $0.006\left(1 / \mathrm{fm}^{3}\right)$.

creases. But contrary to the ideal gas predictions, at a certain density the deuteron to nucleon ratio reaches a maximum and the further density increase leads to a decrease of the $d / n$ ratio. This can be taken into account by the explicit consideration of the specific volumes $V_{i}$ of the fragments ${ }^{27,32}$ for dilute gases, i.e., the fragments are not pointlike but they have a finite volume, thus forming a Van der Waals gas. Generalizing the above mentioned approach $^{32}$ to our case a correction can be obtained to the $a_{i}$ factor of Eq. (8):

$$
\begin{aligned}
a_{i}^{\prime} & =a_{i} \exp \left[-\left(V_{i} N_{i} V_{\mathrm{n}}-Z_{i} V_{\mathrm{p}}\right)\left(n_{i}+n_{\mathrm{n}}+n_{\mathrm{p}}\right)\right] \\
& \simeq a_{i} \exp \left[-\left(V_{i}-N_{i} V_{\mathrm{n}}-Z_{i} V_{\mathrm{p}}\right) n\right] .
\end{aligned}
$$

Owing to this factor the creation of fragments with large volumes (like d) is strongly suppressed, especially at higher densities, thus simulating the quantum mechanical effects in the vicinity of the Mott transition. ${ }^{30,31}$ The volumes $V_{i}$ are taken from Ref. 45 as $V_{i}=2.2,2.2,27.4$, $20.9,22.8$, and $18.1 \mathrm{fm}^{3}$ for $i=\mathrm{p}, \mathrm{n}, \mathrm{d}, \mathrm{t},{ }^{3} \mathrm{He}$, and ${ }^{4} \mathrm{He}$, respectively.

In Fig. 2 the local fragment densities $n_{i}(\overrightarrow{\mathrm{r}})$ for $\mathrm{p}$ and $\alpha$ are shown after the breakup of the nuclear matter. In the central hot region mainly protons are formed, while in the colder side regions (see Fig. 1) the heavier fragments are emitted preferentially.

Once the partial densities and the equilibrium temperature are given, the thermal momentum distributions ${ }_{i}^{1} F_{H}(\overrightarrow{\mathrm{P}}, \overrightarrow{\mathrm{r}})$ in the fluid cell at $\overrightarrow{\mathrm{r}}$ can be written as

$$
\begin{aligned}
{ }_{i}^{1} F_{H}^{\text {cell }}(\overrightarrow{\mathbf{P}}, \overrightarrow{\mathbf{r}}) \equiv & n_{i}(\overrightarrow{\mathbf{r}})\left[2 \pi m_{i} T(\overrightarrow{\mathbf{r}})\right]^{-3 / 2} \\
& \times \exp \left[-\overrightarrow{\mathbf{P}}^{2} / 2 m_{i} T(\overrightarrow{\mathbf{r}})\right] .
\end{aligned}
$$

Since the fluid is moving with the local collective velocity $\vec{v}(\vec{r})$, these distributions should be transformed to the laboratory system by a Lorentz transformation. The differential cross section of particle of type $i$

$$
d \sigma_{i} / d^{3} \overrightarrow{\mathrm{P}} \equiv \int d^{2} b d^{3} r_{i}^{1} F_{H}^{\mathrm{lab}}(\overrightarrow{\mathrm{P}}, \overrightarrow{\mathrm{r}})
$$

is obtained by adding up the contributions of all fluid cells in the laboratory system and then summing up the results 


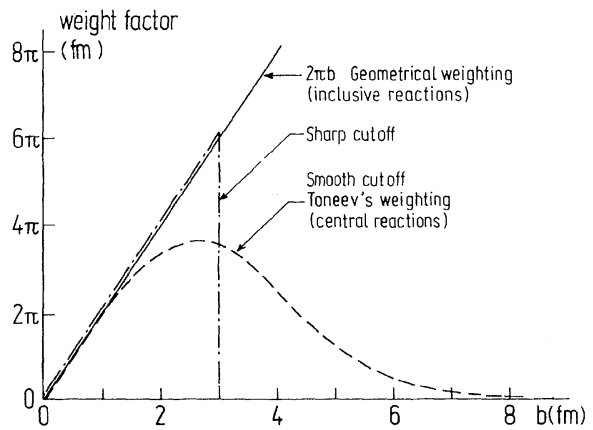

FIG. 3. The central (high multiplicity selected) cross sections are calculated by using the cascade simulations of Toneev (Ref. 47) (dashed line), because collisions with high impact parameters also contribute to the high multiplicity selected data. The sharp cutoff function previously used for the calculation of the contributions of impact parameters in central reaction cross sections in the framework of the hydrodynamic and evaporation model is indicated by the dashed dotted line. The full line shows the impact parameter weighting for inclusive cross sections.

of the different impact parameter calculations weighteci by the corresponding geometrical surfaces.

In previous hydrodynamic model studies it turned out that the applicability of the model in the energy range discussed is restricted to the central and near central collisions of sufficiently massive nuclei. ${ }^{5,42,46}$ In other words, for peripheral collisions or for collisions of small nuclei like $\mathbf{C}+\mathbf{C}$, the model, as a continuum model, is not appropriate. Here the relatively few collisions among the participant nucleons can be followed more accurately in a cascade model. Thus in the following part of the paper we discuss mainly "central" collisions. Within the hydrodynamic model it is not trivial to decide which impact parameters belong to a specific experimental trigger mode, because the fluctuations are not described by the model. In experiments usually a lower bound $M$ for the charged particle multiplicity is required. In the hydrodynamical model the multiplicity decreases monotonically with im- pact parameter and so a lower bound in multiplicity would mean an upper bound $B$ in impact parameter. However, due to fluctuations discussed in the next section, it is possible that collisions with a higher impact parameter than $B$ yield a larger multiplicity than $M$, and smaller impact parameters might contribute to small multiplicity events too. This means that the central, high multiplicity selected data do not correspond to a strict impact parameter range $b=0-B$ but rather to a wider range of impact parameters with the proper weight factors. Inside the hydrodynamical model, however, it is not possible to calculate the portion of collisions at a given impact parameter that contribute to events with multiplicity larger than $M$. We therefore use a cascade simulation of Toneev ${ }^{47}$ where for the $393 \mathrm{MeV} / \mathrm{N} \mathrm{Ne}+\mathrm{U}$ collision the experimental high multiplicity selection ${ }^{48}\left(\boldsymbol{M}_{\text {tot }}>10\right)$ was studied and the contributions of the different impact parameter collisions were determined (Fig. 3). In the following applications we discuss the same reaction and take the given smooth cutoff function of Fig. 3 for the description of central collisions. This procedure is similar to the one used by Schürmann and Chemtob. ${ }^{49}$

\section{MULTIPLICITIES AND THEIR FLUCTUATIONS}

First let us calculate the multiplicities of the various light particles from $\mathrm{p}$ to ${ }^{4} \mathrm{He}$ in the above model (Table I). From these quantities the total charged light particle multiplicities

$$
N_{\text {tot }}=\Sigma_{i=\mathrm{p}^{4}{ }^{4} \mathrm{He}} N_{i}
$$

and the total bound proton multiplicities

$$
N_{B p}=\Sigma_{i=\mathrm{d}-{ }^{4} \mathrm{He}} Z_{i} N_{i}
$$

can be obtained. These values are expectation values obtained under the assumption that the local momentum distributions are equivalent to that of an ideal gas mixture of light nuclear fragments. Even if we neglect the fluctuations arising from this assumption, a lower bound for the fluctuations can be obtained in the following way. We have $Z$ protons in our system and we expect that $N_{F p}$ free

TABLE I. Total light fragment multiplicities for the $\mathrm{Ne}+\mathrm{U}(393 \mathrm{MeV} / \mathrm{N})$ reaction calculated in the hydrodynamic and evaporation model (with $B_{0}=-16 \mathrm{MeV}, K_{0}=200 \mathrm{MeV}$, and $\eta=10 \mathrm{MeV} / \mathrm{fm}^{2} c$ ) at different impact parameters $b$. The average density $(\rho)$, internal energy $(\epsilon)$, and temperature $(T)$ are listed. The quantities $T$ after the breakup do not contain the contribution of the deeply bound fluid cells which form a heavy residue in the model (see Sec. VI).

\begin{tabular}{cccccccccc}
\hline \hline $\begin{array}{c}b \\
(\mathrm{fm})\end{array}$ & $\begin{array}{c}\rho \\
\left(\mathrm{fm}^{-3}\right)\end{array}$ & $\begin{array}{c}\epsilon \\
(\mathrm{MeV} / \mathrm{N})\end{array}$ & $\begin{array}{c}T \\
(\mathrm{MeV})\end{array}$ & $\mathrm{p}$ & $\mathrm{n}$ & $\mathrm{d}$ & $\mathrm{t}$ & ${ }^{3} \mathrm{He}$ & ${ }^{4} \mathrm{He}$ \\
\hline 0 & 0.058 & 3.6 & 10.9 & 10.8 & 38.5 & 6.7 & 26.1 & 2.8 & 24.4 \\
1 & 0.060 & 3.3 & 10.5 & 9.8 & 38.2 & 6.6 & 25.9 & 2.7 & 24.9 \\
2 & 0.060 & 2.6 & 9.6 & 9.0 & 37.6 & 6.1 & 25.1 & 2.4 & 26.0 \\
3 & 0.062 & 1.6 & 8.6 & 8.4 & 37.2 & 5.8 & 21.2 & 2.1 & 27.3 \\
4 & 0.064 & 0.3 & 8.0 & 7.5 & 37.5 & 5.3 & 18.2 & 1.9 & 26.4 \\
5 & 0.066 & -1.1 & 7.4 & 6.5 & 34.6 & 4.7 & 15.6 & 1.6 & 24.9 \\
6 & 0.069 & -2.8 & 6.5 & 5.2 & 30.9 & 3.9 & 12.5 & 1.1 & 23.1 \\
7 & 0.072 & -4.5 & 5.8 & 4.1 & 27.2 & 3.2 & 9.0 & 1.0 & 20.4 \\
8 & 0.076 & -6.0 & 5.5 & 3.3 & 22.3 & 2.6 & 6.7 & 0.7 & 17.0 \\
9 & 0.086 & -7.2 & 4.6 & 2.1 & 18.5 & 1.7 & 5.2 & 0.5 & 15.1 \\
\hline \hline
\end{tabular}


protons are produced in a collision with a given impact parameter and energy. The probability to find a given proton as a free one is

$$
p=N_{F p} / Z,
$$

and the probability to find it in a cluster is

$$
1-p=\left(Z-N_{F p}\right) / Z \text {. }
$$

Therefore the probability to find $n$ free protons is described by the following binomial distribution:

$$
w(n)=p^{n}(1-p)^{Z-n} Z ! /[n !(Z-n) !],
$$

and the expectation value of the fluctuation of the free proton number is $\Delta N_{F p}$,

$$
\begin{aligned}
\left\langle\Delta N_{F p}^{2}\right\rangle & =\left\langle n^{2}\right\rangle-\langle n\rangle^{2}=(1-p)\langle n\rangle \\
& =N_{F p}-\left(Z-N_{F p}\right) / Z .
\end{aligned}
$$

A second source of the fluctuations is the limited sensitivity range of the experimental devices. ${ }^{48}$ Assuming that the fluctuations arising from these two effects are independent, the relative fluctuations can be added to each other:

$$
\begin{aligned}
\left(\Delta N_{F p} / N_{F p}\right)^{\mathrm{obs}}= & \left(\Delta N_{F p} / N_{F p}\right)^{\text {thermodyn }} \\
& +\left(\Delta N_{F p} / N_{F p}\right)^{\text {det }}
\end{aligned}
$$
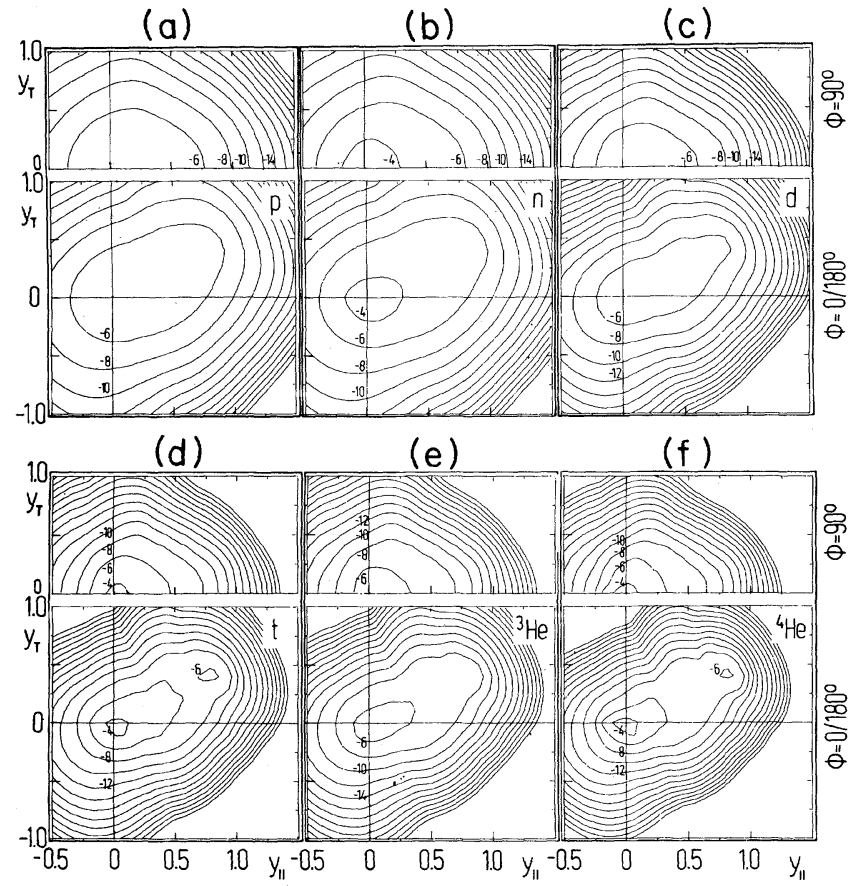

FIG. 4. Contour plots of triple differential invariant cross sections $(1 / p) d^{3} N / d E d \Phi d \cos \theta$ for the reaction ${ }^{20} \mathrm{Ne}(393$ $\mathrm{MeV} / \mathrm{N})+{ }^{238} \mathrm{U}$ at the impact parameter $b=6 \mathrm{fm}$ in the reaction plane $\left(\Phi=0^{\circ} / 180^{\circ}\right)$ and in the plane orthogonal to it $\left(\Phi=90^{\circ}\right)$. The contour lines labeled by the parameter $q$ correspond to a value of $10^{q} /\left(\mathrm{sr} \mathrm{MeV}^{2}\right)$. Parts (a), (b), (c), (d), (e), and (f) correspond to $\mathrm{p}, \mathrm{n}, \mathrm{d}, \mathrm{t},{ }^{3} \mathrm{He}$, and ${ }^{4} \mathrm{He}$ cross sections, respectively. The bounceoff effect is predominantly observable in $t$, ${ }^{3} \mathrm{He}$, and ${ }^{4} \mathrm{He}$ spectra.

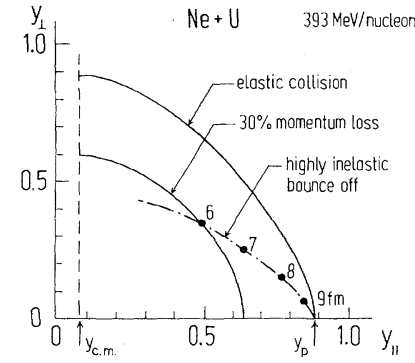

FIG. 5. The dependence of the c.m. bounceoff deflection angle and inelasticity of the impact parameter $b$. At impact parameters lower than $3 \mathrm{fm}$ the second local maximum of the spectrum vanishes and so the inelasticity cannot be uniquely determined, but the bounceoff angle is measurable.

Since after the collision $Q$ free nucleons are produced and one nucleon is observed by the detector with a probability $q$, the total observable fluctuation can be estimated as

$$
\Delta N_{F p}^{\mathrm{obs}}=q \sqrt{(1-p) p Z}+\sqrt{(1-q) q p Z},
$$

where the first term describes the thermal fluctuations and the second one the limited sensitivity of the detector. The calculated energy spectra may provide us with a possibility to estimate roughly the detector sensitivity for a given reaction.

\section{DIFFERENTIAL CROSS SECTIONS AND CORRELATIONS}

Calculations were performed for ${ }^{20} \mathrm{Ne}+{ }^{238} \mathrm{U}$ collisions at a projectile energy of $393 \mathrm{MeV} /$ nucleon. In the triple differential cross sections strong azimuthal correlations are obtained at $\Delta \Phi=180^{\circ}$. The two main jets arise from the target and projectile evaporations, and can be observed as local peaks (Fig. 4) in the cross section at a fixed impact parameter. The two peaks are approximately $\Delta \phi=180^{\circ}$ from each other in the nucleus-nucleus c.m. frame because of momentum conservation. From the position of the peaks in the c.m. momentum space the momentum lost in the inelastic collision can be determined. Because of the lower temperatures of the projectile and target remnants at the breakup moment (Fig. 1), mainly heavier bound fragments, e.g., ${ }^{4} \mathrm{He}$ (Fig. 2), are formed here. Furthermore, because these are in thermal equilibrium with other species, their thermal velocities are considerably smaller than those of the lighter species and so the smearing due to the random thermal velocities is weaker while the collective velocities are the same. Thus the heavier fragment cross sections show the collective flow properties more clearly (Fig. 4), so that the deflection angle and the loss of collective momentum in the c.m. system are accurately measurable using the triple differential ${ }^{4} \mathrm{He}$ cross sections (Fig. 5).

Experimental triple differential (azimuth dependent) cross sections are not available yet, but $p, d$, and $t$ double differential cross sections for central $\left(\boldsymbol{M}_{\text {tot }}>10\right) \mathrm{Ne}(393$ $\mathrm{MeV} / \mathrm{N})+\mathrm{U}$ reactions were measured recently. ${ }^{2,3}$ Averaging over the azimuth angle in the triple differential 


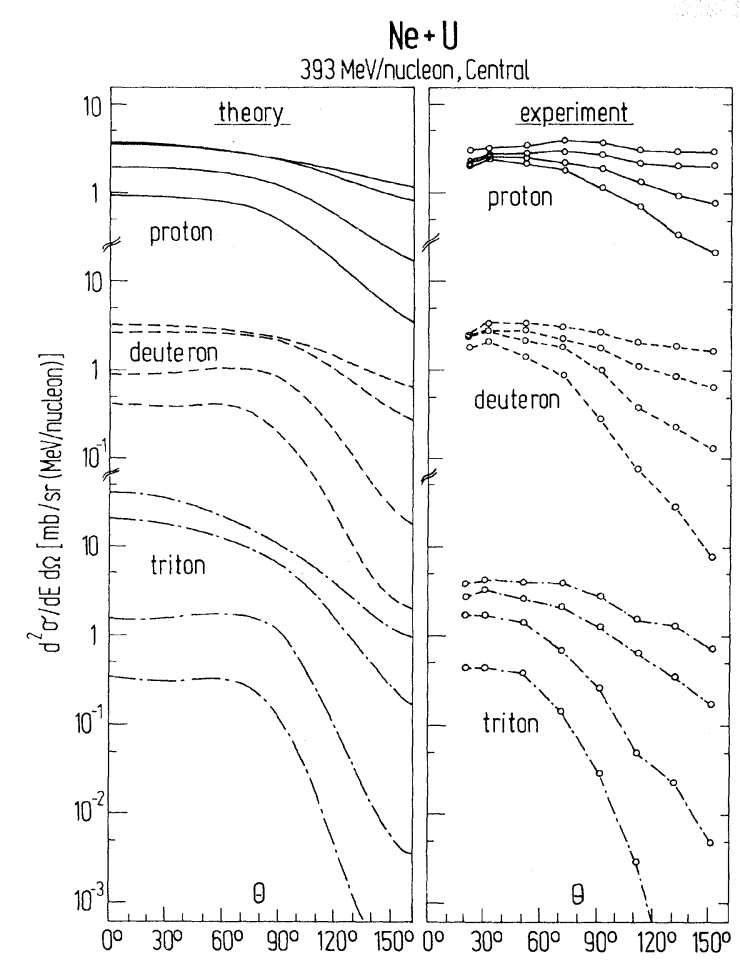

FIG. 6. Double differential $\mathrm{p}, \mathrm{d}$, and $\mathrm{t}$ cross sections for the central $\mathrm{Ne}(393 \mathrm{MeV} / \mathrm{N})+\mathrm{U}$ reaction. The experimental cross sections (Refs. 2 and 3) (points) are at 12, 21, 47, and $82 \mathrm{MeV} / \mathrm{N}$ energies and the calculated ones at $10,20,50$, and $80 \mathrm{MeV} / \mathrm{N}$.

cross sections given in Fig. 4 and integrating over $b$ with the smooth cutoff (Fig. 3), we obtain the double differential cross sections in our model. The comparison to the experimental data shows an overall agreement with discrepancies remaining in some regions (Fig. 6). At small angles $\left(\theta=20^{\circ}-30^{\circ}\right)$ the energy dependence in our calculation is stronger and the sideward peaking is predicted at higher angles. Both of these deviations indicate that in

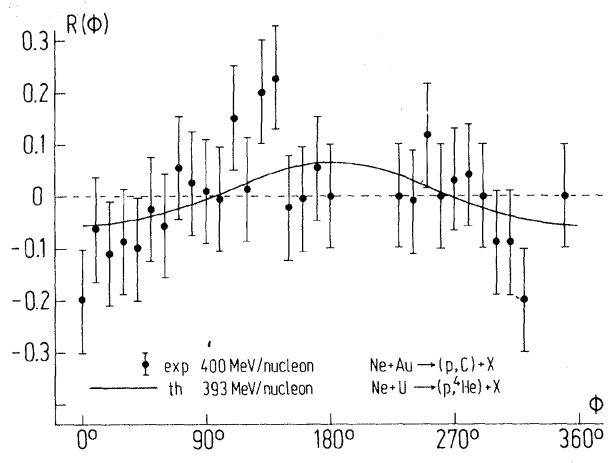

FIG. 7. Heavy-light fragment correlations calculated in the theoretical model for one impact parameter $b=6 \mathrm{fm}$. The heavy fragment is ${ }^{4} \mathrm{He}$ at $\theta=90^{\circ}$ in the laboratory system measured in coincidence with a proton at $\theta=40^{\circ}$ in the energy range $30-50 \mathrm{MeV}$. The correlation at $\Delta \Phi=180^{\circ}$ is the consequence of the bounceoff effect. The experimental points are taken from Ref. 4.

the experiment the collisions with higher impact parameters have a larger weight than assumed by the smooth cutoff curve, or that the one fluid model somewhat overestimates the bounce-off angles at a given impact parameter. However, the forward suppression and sidewards peaking in the experimental and calculated data indicate the presence of the collective bounceoff process. Earlier speculations that the forward suppression in $p$ spectra is caused by the formation of composite fragments does not hold, because the composite fragment cross sections $(d, t)$ are also suppressed in forward directions both in the experiment and in our calculation.

Recently azimuthal correlations between slow heavy and fast light fragments have also been measured. ${ }^{4}$ The results show strong $180^{\circ}$ azimuthal correlations, providing further experimental evidence for collective processes. In our model the correlation function ${ }^{4} R(\Phi)$ is defined on the basis of the triple differential cross section as

$$
R(\delta)=\frac{2 \pi \int \sigma_{{ }_{4} \mathrm{He}}\left(90^{\circ}, \phi, \epsilon\right) \sigma_{\mathrm{p}}\left(40^{\circ}, \phi+\delta, 30-40 \mathrm{MeV}\right) d \phi d \epsilon}{\int \sigma_{{ }^{4} \mathrm{He}}\left(90^{\circ}, \phi, \epsilon\right) d \phi d \epsilon \int \sigma_{\mathrm{p}}\left(40^{\circ}, \phi+\delta, 30-40 \mathrm{MeV}\right) d \phi}-1
$$

where $\sigma_{i}$ denotes the triple differential $i$ particle cross section defined by Eq. (13):

$$
\sigma_{i}(\overrightarrow{\mathbf{P}}) \equiv d \sigma_{i} / d^{3} \overrightarrow{\mathbf{P}}
$$

The experimentally observed $180^{\circ}$ azimuthal correlations are in qualitative agreement with the results of the calculations (Fig. 7). In our calculation this effect is caused by the collective bounceoff: The heavier fragments $\left({ }^{4} \mathrm{He}\right)$ are produced mainly on the colder target side and the protons on the opposite side. This $\Phi=180^{\circ}$ correlation between protons and heavy nuclei holds also for heavier fragments (target residues described in Sec. VI).

\section{FORMATION OF HEAVY FRAGMENTS \\ IN THE FRAMEWORK OF HYDRODYNAMICS}

In the preceding sections only the light fragment formation was discussed under the assumption that there is local thermal and chemical equilibrium between these fragments. However, this assumption is satisfactory only for the regions where we have a higher excitation energy at the breakup phase. There are other regions (mainly the "target residue") where the nucleons are bound deeper than $-8 \mathrm{MeV}$, and so light fragment formation is not possible. The fluids belonging to this deeply bound region are not taken into account in the calculation of the light fragment cross sections. This region is of considerable size compared to the whole reaction zone so that we have to choose another description other than that in the previous sections, since local equilibrium assumptions may not be applied.

In the most typical intermediate impact parameter region $10-30 \%$ of the fluid cells form a connected spatial region where the nucleons are deeply bound at breakup. We assume that the nucleons in this region form an inter- 
mediate nucleus with given mass and excitation energy. The mass of the nucleus is

$$
A_{\text {bnd }}=\int_{V_{\text {bnd }}} d^{3} \overrightarrow{\mathrm{r}} n(\overrightarrow{\mathrm{r}})
$$

where the integral runs over the fluid volume $V_{\text {bnd }}$ where the internal excitation energy $\epsilon<-8 \mathrm{MeV} /$ nucleon. At the calculation of the total excitation energy of the heavy fragment we take into account the internal energy $\epsilon$ and the energy arising from the spread of the flow momenta in this region. We integrate the four-momenta of the fluid cells $p(\overrightarrow{\mathrm{r}})=[\overrightarrow{\mathrm{p}}(\overrightarrow{\mathrm{r}}), w(\overrightarrow{\mathrm{r}})]$,

$$
\begin{aligned}
\overrightarrow{\mathbf{P}} & =\int d^{3} \overrightarrow{\mathrm{r}} \overrightarrow{\mathrm{p}}(\overrightarrow{\mathrm{r}}) n(\overrightarrow{\mathrm{r}}) \\
& =\int d^{3} \overrightarrow{\mathrm{r}} n(\overrightarrow{\mathrm{r}})\left[m_{n}+\epsilon(\overrightarrow{\mathrm{r}})\right] \gamma(\overrightarrow{\mathrm{r}}) \overrightarrow{\mathrm{v}}(\overrightarrow{\mathrm{r}}), \\
W & =\int d^{3} \overrightarrow{\mathrm{r}} w(\overrightarrow{\mathbf{r}}) n(\overrightarrow{\mathrm{r}}) \\
& =\int d^{3} \overrightarrow{\mathrm{r}} n(\overrightarrow{\mathrm{r}})\left[m_{n}+\epsilon(\overrightarrow{\mathrm{r}})\right] \gamma(\overrightarrow{\mathrm{r}}),
\end{aligned}
$$

where

$$
\gamma(\overrightarrow{\mathrm{r}})=1 / \sqrt{\left[1-v(\overrightarrow{\mathrm{r}})^{2}\right]} .
$$

In this way we can get the rest mass of the heavy fragment as

$$
M_{0}=\sqrt{\left(W^{2}-\overrightarrow{\mathbf{P}}^{2}\right)},
$$

and its specific excitation energy above the ground state $(-8 \mathrm{MeV})$ :

$$
E^{*}=\left(M_{0}-m_{n} A_{\text {bnd }}\right) / A_{\text {bnd }}+8 \mathrm{MeV} / \mathrm{N} .
$$

This heavy fragment is expected to be emitted with a recoil energy $E_{R}$ corresponding to the velocity

$$
\vec{\beta}=\overrightarrow{\mathbf{P}} / W \text {. }
$$

Thus we can get a rough estimate of the properties of the created heavy residue. The underlying basic idea is very similar to the abrasion-ablation model. ${ }^{50}$ However, we are not bound to the straight line geometry, the recoil has a transverse component, and we can determine the excitation energy of the residue without any additional assumption. We neglected, however, certain processes, e.g., the excitation stemming from the deformation of the spatial region where the deeply bound nucleons are situated at the breakup moment and the excitation arising from the sharp cutoff of the density at the surface. Thus we underestimate the excitation energy by a few $\mathrm{MeV} / \mathrm{N}$. On the other hand, owing to the rough grid, the accuracy of the predicted mass is of the order of the mass contained in the fluid cells at the surface of the deeply bound region, which form a layer of width $\simeq \Delta x / 2$. This yields an essential relative error, especially for smaller residues:

$$
\Delta A / A \simeq(9 \pi n / 2 A)^{1 / 3}, \Delta x / 2 \simeq 0.67 A^{-1 / 3} .
$$

Such excited target residues have been found recently in ${ }^{12} \mathrm{C}(84 \mathrm{MeV} / \mathrm{N})+$ induced reactions ${ }^{51-52}$ around $A \simeq 50$ $(\Delta A \simeq 10)$ with a recoil energy of about $1 \mathrm{MeV} / \mathrm{N}$ $(\beta \simeq 0.04-0.05 c)$. The small spread of the mass spectrum indicates that these are not fission products, and evaporation calculations show that these final states may arise
TABLE II. Parameters of the intermediate excited target residues of mass larger than 10 for the $\mathrm{Ne}+\mathrm{U}(393 \mathrm{MeV} / \mathrm{N})$ reaction calculated in the hydrodynamic and evaporation model $\left(B_{0}=-16 \mathrm{MeV}, K_{0}=200 \mathrm{MeV}\right.$, and $\left.\eta=10 \mathrm{MeV} / \mathrm{fm}^{2} c\right)$ at different impact parameters $b$. The expected mass $A_{\text {bnd }}$, recoil angle $\theta$, recoil energy $E_{R}$, excitation energy $E^{*}$ (without surface and deformation energies), and recoil velocity $\beta$ are listed.

\begin{tabular}{cccccc}
\hline \hline $\begin{array}{c}b \\
(\mathbf{f m})\end{array}$ & $A_{\text {bnd }}$ & $\begin{array}{c}\theta \\
(\mathrm{deg})\end{array}$ & $\begin{array}{c}E_{R} \\
(\mathrm{MeV})\end{array}$ & $\begin{array}{c}E^{*} \\
(\mathrm{MeV} / \mathrm{N})\end{array}$ & $\beta$ \\
\hline 4 & 24 & 84 & 34 & -0.9 & 0.055 \\
5 & 44 & 83 & 37 & -1.7 & 0.043 \\
6 & 68 & 81 & 37 & -1.9 & 0.034 \\
7 & 97 & 81 & 29 & -2.2 & 0.025 \\
8 & 129 & 82 & 19 & -2.4 & 0.018 \\
9 & 148 & 90 & 8 & -2.6 & 0.011 \\
\hline \hline
\end{tabular}

from an excited intermediate compound nucleus of $A \simeq 90$ and $E^{*} \simeq 8 \mathrm{MeV} / \mathrm{N}$.

At $393 \mathrm{MeV} / \mathrm{N}$ projectile energy the residues belonging to the lowest impact parameters have high excitation energy so that they probably cannot be observed as one heavy residue (Table II). At high impact parameters the fluctuations and final state interaction effects are large compared to our recoil energy so that our estimated recoil angles and energies are not easily observable. Nevertheless, in an intermediate impact parameter region there should be a correlation between the residue mass and the mean recoil angle.

In Table II the mass, excitation energy, and emission angle of the expected heavy residue are shown versus the impact parameter. In a wide impact parameter range we get an intermediate nucleus with relatively small (a few $\mathrm{MeV} /$ nucleon) excitation energy. These intermediate states may decay by light fragment or nucleon emission to yield a somewhat lighter observable heavy fragment. These final fragment states will be in the vicinity of the intermediate state on the $(N, Z)$ plane. In Fig. 8 the inter-

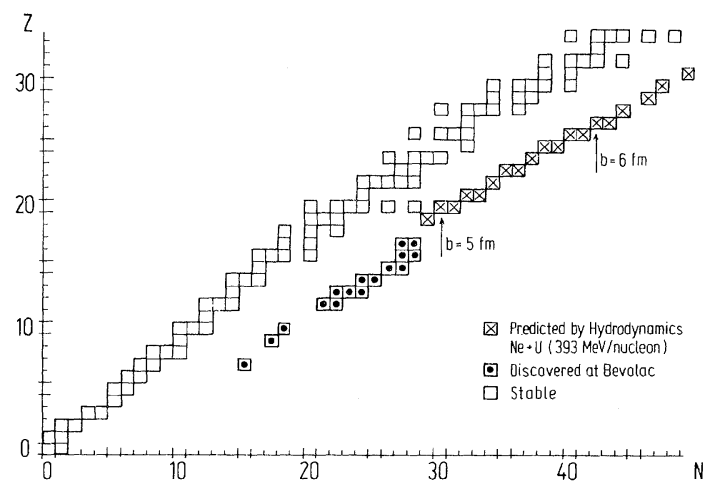

FIG. 8. The estimated position of the intermediate compound target residue on the $(N, Z)$ plane from the hydrodynamic model. The $N$ to $Z$ ratio is that of uranium. The neutron rich excited heavy residues can decay mainly by $\mathrm{p}, \mathrm{n}$, and $\alpha$ emission and so the final states are in a narrow region $(\Delta A \simeq 4-6)$ around the indicated line. In this way neutron rich isotopes might be produced. 
mediate residues are shown on the $(N, Z)$ plane for several impact parameters in a $\mathrm{Ne}+\mathrm{U}$ reaction at 393 $\mathrm{MeV} /$ nucleon bombarding energy. These results indicate that in reactions with heavy target or projectile new heavier neutron rich isotopes might be produced similarly to the already observed lighter ones arising from projectile fragmentation. ${ }^{53}$

The model of the residue formation presented here does not work at small and at very large impact parameters. At small impact parameters the intermediate residue has a large excitation energy. This is caused by the fact that the deeply bound cells do not occupy a singly connected spatial region, but rather a ring or two or more separate connected regions. The flow velocity differences between different parts of the deeply bound region become large and this is the reason for the apparent higher excitation energies. In this case the intermediate residue fissions into two or more parts or it may be that already at the breakup moment the deeply bound cells form several fragments. The latter happens at large impact parameters also, where both target and projectile residues are present. However, for these large impact parameters the participant zone is already very small and contains only a few nucleons, so that the hydrodynamic description loses its validity and other processes such as microscopic $\mathbf{n}-\mathbf{n}$ correlations become more important. , $^{46}$

\section{EVENT BY EVENT ANALYSIS}

Recently, for the investigation of the possible collective properties, several methods were introduced such as the sphericity tensor, energy flow tensor, and thrust analyses. ${ }^{54-58}$ The first experimental studies with the plastic ball $^{48}$ and with the streamer chamber ${ }^{59}$ are in progress.

Theoretically it is straightforward to evaluate the real symmetric sphericity matrix in the c.m. frame:

$$
M_{\alpha \beta}=\Sigma_{i} w_{i} p_{i \alpha} p_{i \beta}, \quad \alpha, \beta=x, y, z,
$$

where $i$ runs over all emitted charged particles (up to ${ }^{4} \mathrm{He}$ for the plastic ball experiments and in our calculations), and $w_{i}$ is a weight factor which can depend on the type of particle $i$. In the case of the energy flow tensor analysis $w_{i}=1 / 2 m_{i}$. The eigenvalues $Q_{i}$ and eigenvectors $\overrightarrow{\mathrm{e}}_{1}, \overrightarrow{\mathrm{e}}_{2}, \overrightarrow{\mathrm{e}}_{3}$ of the tensor can be determined. If we normalize the sum of eigenvalues to unity so that $Q_{3}>Q_{2}>Q_{1}$ we can evaluate the commonly used quantities: sphericity ${ }^{54}$ $S=1.5\left(Q_{1}+Q_{2}\right)$, flatness $F=\sqrt{3}\left(Q_{2}-Q_{1}\right) / 2$, jet angle $\theta_{\text {c.m. }}=\arccos \left(\left[\overrightarrow{\mathrm{e}}_{3}\right]_{z} / e_{3}\right)$, and aspect $\operatorname{ratios}^{58} R_{1}=Q_{3} / Q_{1}$ and $R_{2}=Q_{2} / Q_{1}$.

Before discussing the calculational details let us emphasize two problems. Most detectors do not detect neutrons, and these need not have the same distribution as the average of the other light charged fragments. Similarly, heavy clusters and residues are also not detected. Therefore, to gain a result which is comparable to experiments the analysis of the cluster formation is unavoidable.

From the final momentum distribution of the light fragments ${ }_{j}^{1} F_{H}$ [see Sec. III, Eqs. (12) and (13)] the sphericity tensor can be obtained as

$$
\begin{aligned}
M_{\alpha \beta}=\int & d^{3} \overrightarrow{\mathrm{r}} \Sigma_{j_{\text {clust }}} w_{j} \\
& \times \int d^{3} \overrightarrow{\mathbf{P}}^{\text {c.m. } \cdot\left[{ }_{j}^{1} F_{H}^{\text {c.m. }}\left(\overrightarrow{\mathbf{P}}^{\text {c.m. }}, \overrightarrow{\mathrm{r}}\right)\right] P_{j \alpha}^{\text {c.m. }} P_{j \beta}^{\text {c.m. }}} .
\end{aligned}
$$

Expression (28) can be simplified ${ }^{60}$ and separated into a thermal and a flow term if we use Galilei transformation for the determination of c.m. momentum distribution from the local rest frames of the cells instead of a Lorentz transformation. The importance of such a thermal term arising from this separation is discussed in Ref. 60 .

In Fig. 9 the jet angle and the $R_{1}$ aspect ratios are shown for different impact parameters for protons, alpha particles, and the sum of all light charged fragments up to ${ }^{4} \mathrm{He}$, in an energy flow tensor analysis $\left(w_{i}=1 / 2 m_{i}\right)$, and in global analysis with $w_{i}=1$. We have seen in the angular distributions that the ${ }^{4} \mathrm{He}$ particles show the collective jet structure stronger than the protons. Note the difference of proton and alpha aspect ratios at small and large impact parameters.

At small impact parameters $b \simeq 0$ the alphas are produced mainly in an outer ring yielding a very flat oblate flow tensor. $\overrightarrow{\mathrm{e}}_{1}$ points in the $z$ direction, $\overrightarrow{\mathrm{e}}_{3}$ is orthogonal to the reaction plane up to $b=3 \mathrm{fm}$, and $Q_{3} \simeq Q_{2}>Q_{1}$. As the impact parameter increases the ring of colder matter first rotates then disappears and the target residue region will contain the colder matter where the alphas are emitted from. Now the differences between the eigen-

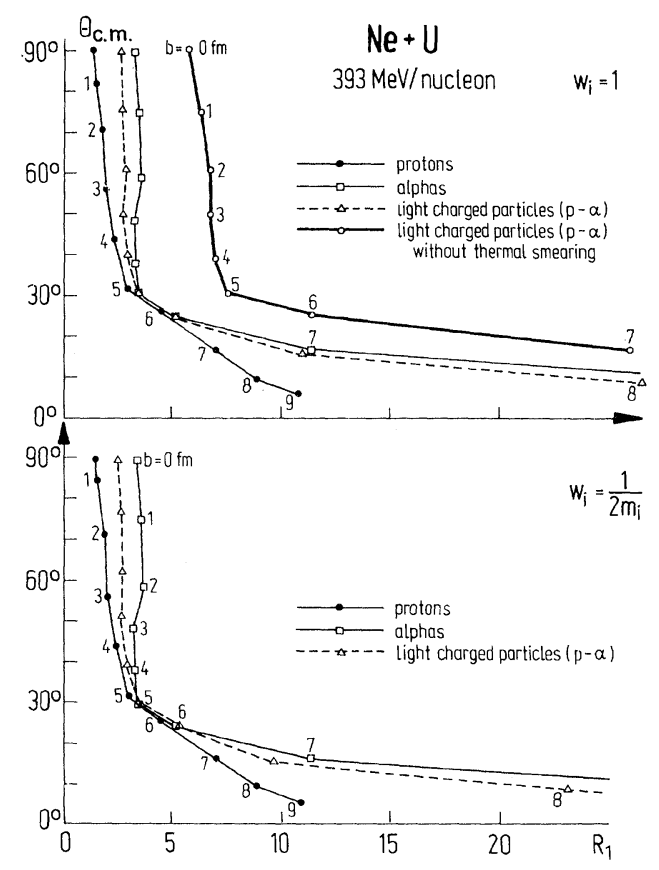

FIG. 9. Calculated $R_{1}$ aspect ratios versus the jet angle $\theta_{\text {c.m. }}$. for different impact parameters in the $\mathrm{Ne}(393 \mathrm{MeV} / \mathrm{N})+\mathrm{U}$ reaction plotted separately for protons $\mathrm{p}$ (dots), alpha particles $\alpha$ (open squares), and all charged light fragments (open triangles). The lines indicated by open circles represent light particles without thermal smearing. Two different weights $w_{i}=1, \frac{1}{2} m_{i}$ are compared. 
values of the alpha particle flow tensor decrease and the alphas can even be emitted slightly more spherically than at small impact parameters. The $R_{1}$ aspect ratio for alphas starts to grow rapidly when $b>R_{\mathrm{t}}-R_{\mathrm{p}} \simeq 4 \mathrm{fm}$, and $\overrightarrow{\mathrm{e}}_{3}$ starts to point in the direction of the bounced off projectile inside the reaction plane $(y, z)$. Now the difference between the proton and alpha flow tensor is luminous; the alphas are formed in the target and projectile spectator regions, far from each other in the phase space (Fig. 2). $\overrightarrow{\mathrm{e}}_{3}$ is in the reaction plane and $Q_{3} \gg Q_{2}$.

The proton flow tensor changes continuously; $\overrightarrow{\mathrm{e}}_{3}$ is always in the reaction plane and points in the direction of the bounced off projectile. The flow angle $\theta_{\text {c.m. }}$ and $R_{1}$ change monotonically with increasing impact parameter.

Due to these systematics the $R_{1}(\theta)$ function shows a very sharp change for alpha particles according to our calculations (Fig. 9). Such structure can be expected only in asymmetric collisions like $\mathrm{Ne}+\mathrm{U}$; however, statistical fluctuations may wash out this structure, especially in the case of alpha particles owing to their low multiplicity.

In Fig. 9 the $R_{1}$ parameters of the emitted light fragments calculated with and without the thermal momenta are also compared to each other. The thermal smearing increases the sphericity and decreases $R_{1}$ by $20-30 \%$ at all impact parameters. This indicates that in earlier theoretical global flow analyses of Ref. 56 the neglect of thermal smearing effects leads to an overestimation of the thrust value.

In a comparison of our calculations with the experiments some difficulties arise. The limited sensitivity of the detector in momentum space and the fact that most detectors do not cover a spherically symmetric region around the c.m. in the momentum space cause serious problems. The sphericity matrix detected by a given detector is not equal to the one defined by Eq. (28), but rather is given by

$$
\begin{aligned}
M_{\alpha \beta}=\int & d^{3} \overrightarrow{\mathrm{r}} \Sigma_{j_{\text {clust }}} w_{j} \\
& \times \int_{\mu_{\text {det }}} d^{3} \overrightarrow{\mathbf{P}}^{\text {c.m. }} \cdot\left[{ }_{j}^{1} F^{\text {c.m. }} \cdot\left(\overrightarrow{\mathbf{P}}^{\text {c.m. }}, \overrightarrow{\mathrm{r}}\right)\right] P_{j \alpha}^{\text {c.m. }} \cdot P_{j \beta}^{\text {c.m. }},
\end{aligned}
$$

where $\mu_{\text {det }}$ is the sensitivity region of the detector in the momentum space. Unfortunately, owing to the separation introduced $^{60}$ we are not able to easily reproduce such a restriction.

Figure 10 might provide, however, an insight into this problem. The rapidity distribution of the alpha particles is shown in the reaction plane for a $\mathrm{Ne}(393 \mathrm{MeV} / \mathrm{N})+\mathrm{U}$ calculation together with the acceptance of the plastic ball. ${ }^{48}$ The local maximum of the cross section caused by the evaporation of the bounced off projectile lies at the upper energy limit of the plastic ball for full particle identification $(\simeq 250-300 \mathrm{MeV} / \mathrm{N})$ owing to the fact that the collision is highly inelastic. The better the peaked structure of the cross section shows up in the plastic ball observables, the closer the projectile energy is to this upper energy limit (i.e., at $300-400 \mathrm{MeV} / \mathrm{N}$ ). At very high projectile energies, in the $\mathrm{GeV} / \mathrm{N}$ region, the fluctuations and

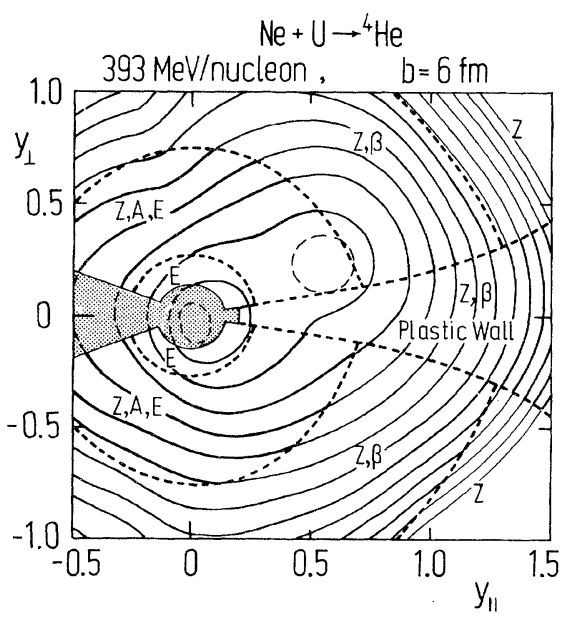

FIG. 10. Contour plot of the calculated triple differential invariant ${ }^{4} \mathrm{He}$ cross section for the $\mathrm{Ne}(393 \mathrm{MeV} / \mathrm{N})+\mathrm{U}$ reaction at the impact parameter $b=6 \mathrm{fm}$. Two peaks arise from the target and projectile evaporations. The dashed lines indicate the plastic ball and plastic wall response taken from Ref. 48. The full determination of particles is possible in the region indicated by $(Z, A, E)$ so that the experimental determination of the matrix $M$ is restricted to this region. The energy flow matrix (Ref. 58) can be determined, however, by using the information from the interior layer, labeled $E$, too. The maximum arising from the projectile evaporation is detectable in the above reaction by the plastic ball (Ref. 48).

the effects arising from the special sensitivity range of the detector are probably too strong to allow for a determination of the collective flow variables.

Because of these difficulties, a Monte Carlo simulation would be extremely useful where for some previously given emission patterns the sphericity and jet angle variables were evaluated in exactly the same way and with all restrictions as was done with the actual experimental device.

\section{CONCLUSIONS}

We have presented results of an extended fluiddynamical model which incorporated the formation of light nuclear fragments and their final thermal evaporation. As we have seen in the preceding sections there are numerous signatures of the collective flow processes observable in the fragment emission such as (i) the sidewards peaked structure of the double differential cross sections, especially for composite fragments; (ii) the correlations arising from the collective structure of the triple differential cross sections both between two protons, ${ }^{5,46}$ and light and heavy fragments; and (iii) the large jet angles in the global analysis of the central events. These experimental signatures provide evidence of the existence of a collective flow of the hot, dense nuclear matter formed in relativistic heavy ion collisions. ${ }^{61}$ These collective processes are governed by the underlying equation of state and trans- 
port properties of the nuclear, hadronic, or quark matter. The detailed study of the composite fragment cross sections in $4 \pi$ exclusive experiments to be completed in the near future may provide a unique tool for the determination of the properties of strongly interacting matter at extreme conditions and may allow for a determination of phase transitions in dense nuclear matter. ${ }^{62}$ We are looking forward to the analysis of $4 \pi$ experiments presently underway.

\section{ACKNOWLEDGMENTS}

Enlightening discussions with H. H. Gutbrod and H. G. Ritter are gratefully acknowledged. The authors thank $\mathrm{V}$. D. Toneev for communication of results prior to publication. This work was supported by the Alexander von Humboldt Stiftung, by the Bundesministerium für Forschung und Technologie, and by the Gesellschaft für Schwerionenforschung.
*Present address: Michigan State University, East Lansing, MI 48823.

†Present address: University of Madras, Madras, India.

${ }^{1}$ H. G. Baumgardt, J. U. Schott, Y. Sakamoto, E. Schopper, H. Stöcker, J. Hofmann, W. Scheid, and W. Greiner, Z. Phys. A 273, 359 (1975); J. Hofmann, H. Stöcker, U. Heinz, W. Scheid, and W. Greiner, Phys. Rev. Lett. 36, 88 (1976); H. G. Baumgardt and E. Schopper, J. Phys. Lett. G5, L231 (1979).

${ }^{2}$ R. Stock, H. H. Gutbrod, W. G. Meyer, A. M. Poskanzer, A. Sandoval, J. Gosset, C. H. King, G. King, Ch. Lukner, Nguyen Van Sen, G. D. Westfall, and K. L. Wolf, Phys. Rev. Lett. 44, 1243 (1980).

${ }^{3}$ H. H. Gutbrod, in Proceedings of the Symposium on High Energy Nuclear Interactions, Hakone, Japan, edited by $\mathbf{K}$. Nakai and A. S. Goldhaber, 1980, Lawrence Berkeley Laboratory Report LBL-11123, 1980, p. 93.

${ }^{4}$ W. G. Meyer, H. H. Gutbrod, Ch. Lukner, and A. Sandoval, Phys. Rev. C $\underline{22}, 179$ (1980).

${ }^{5}$ L. P. Csernai, W. Greiner, H. Stöcker, I. Tanihata, S. Nagamiya, and J. Knoll, Phys. Rev. C 25 , 2482 (1982); I. Tanihata, see Ref. 3, p. 382.

${ }^{6}$ L. P. Csernai and H. W. Barz, Z. Phys. A 296, 173 (1980).

${ }^{7} \mathbf{J}$. Hofmann, W. Scheid, and W. Greiner, Nuovo Cimento $33 \mathrm{~A}$, 343 (1976); J. A. Maruhn, T. A. Welton, and C. Y. Wong, J. Comput. Phys. 20, 326 (1976); J. Hofmann, H. Stöcker, U. Heinz, W. Scheid, and W. Greiner, Phys. Rev. Lett. $\underline{36}, 88$ (1976); J. A. Maruhn, in Proceedings of the Topical Conference on Heavy Ion Collisions, Fall Creek Falls State Park, Tennessee, 1976, p. 156.

${ }^{8}$ A. A. Amsden, G. F. Bertsch, F. H. Harlow, and J. R. Nix, Phys. Rev. Lett. 35, 905 (1975); A. A. Amsden, F. H. Harlow, and J. R. Nix, Phys. Rev. C 15, 2059 (1977); A. A. Amsden et al., Phys. Rev. Lett. 38, 1155 (1977); J. R. Nix and D. Strottman, Phys. Rev. C 23, 2548 (1981).

${ }^{9}$ H. Stöcker, W. Greiner, and W. Scheid, Z. Phys. A 286, 121 (1978); H. Stöcker, J. A. Maruhn, and W. Greiner, ibid. 290, 297 (1979); H. Stöcker, R. Y. Cusson, J. A. Maruhn, and W. Greiner, ibid. 294, 125 (1980); H. Stöcker, J. Hofmann, J. A. Maruhn, and W. Greiner, Prog. Part. Nucl. Phys. 4, 125 (1980).

10P. Danielewicz, Nucl. Phys. A314, 465 (1979).

${ }^{11}$ L. P. Csernai, B. Lukács, and J. Zimányi, Lett. Nuovo Cimento $\underline{27}, 111$ (1980).

${ }^{12}$ H. H. Tang and C. Y. Wong, Phys. Rev. C 21, 1846 (1980).

${ }^{13}$ G. Buchwald, L. P. Csernai, J. Maruhn, W. Greiner, and H. Stöcker, Phys. Rev. C 24, 135 (1981); G. Buchwald, L. P. Csernai, G. Graebner, J. Maruhn, W. Greiner, and H. Stöcker, Z. Phys. A $\underline{303}, 111$ (1981).

${ }^{14}$ H. Stöcker, J. Maruhn, and W. Greiner, Phys. Rev. Lett. 44, 725 (1980); Z. Phys. A 293, 173 (1979).
${ }^{15}$ H. Stöcker, L. P. Csernai, G. Graebner, G. Buchwald, H. Kruse, R. Y. Cusson, J. A. Maruhn, and W. Greiner, Phys. Rev. C 25, 1873 (1982); H. Stöcker et al., Phys. Rev. Lett. 47, 1807 (1981).

${ }^{16}$ A. J. Sierk and J. R. Nix, Phys. Rev. C 22 , 1920 (1980); I. N. Mishustin and L. M. Satarov, Yad. Fiz. 37, 894 (1983) [Sov. J. Nucl. Phys. (to be published)].

${ }^{17}$ J. I. Kapusta and D. Strottman, Phys. Rev. C 23, 1282 (1981).

${ }^{18}$ G. F. Bertsch and A. A. Amsden, Phys. Rev. C 18, 1293 (1978).

${ }^{19}$ G. Westfall, J. Gosset, P. J. Johansen, A. M. Poskanzer, W. G. Meyer, H. H. Gutbrod, A. Sandoval, and R. Stock, Phys. Rev. Lett. 37, 1202 (1976); J. Gosset, H. H. Gutbrod, W. G. Meyer, A. M. Poskanzer, A. Sandoval, R. Stock, and G. Westfall, Phys. Rev. C 16, 629 (1977).

${ }^{20}$ J. Gosset, J. I. Kapusta, and D. Westfall, Phys. Rev. C $\underline{18}, 844$ (1978).

${ }^{21}$ I. Tanihata, S. Nagimaya, S. Schnetzer, and H. Steiner, Phys. Lett. 100B, 121 (1981).

22J. D. Stevenson, J. Martins, and P. B. Price, Phys. Rev. Lett. 47, 990 (1981).

${ }^{23}$ S. Nagamiya, M.-C. Lemaire, E. Moeller, S. Schnetzer, G. Shapiro, H. Steiner, and I. Tanihata, Phys. Rev. C 24, 971 (1981).

${ }^{24}$ Gy. Fái and J. Randrup, Nucl. Phys. A381, 557 (1982); J. Randrup and S. E. Koonin, ibid. A356, 223 (1981).

${ }^{25}$ P. J. Siemens and J. I. Kapusta, Phys. Rev. Lett. 43, 1486 (1979).

${ }^{26}$ A. Z. Mekjian, Phys. Rev. Lett. $\underline{38}, 640$ (1977); Phys. Rev. C 17, 1051 (1978); Nucl. Phys. A312, 491 (1978).

${ }^{27}$ P. R. Subramanian, L. P. Csernai, H. Stöcker, J. A. Maruhn, W. Greiner, and H. Kruse, J. Phys. G 7, 1241 (1981).

${ }^{28}$ S. Das Gupta and A. Z. Mekjian, Phys. Rep. 72, 131 (1981).

${ }^{29}$ J. I. Kapusta, Phys. Rev. C 16, 1493 (1977).

${ }^{30}$ G. Röpke, L. Münchow, and H. Schulz, Phys. Lett. 110B, 21 (1982).

${ }^{31}$ J. Knoll, L. Münchow, G. Röpke, and H. Schulz, Phys. Lett. 112B, 13 (1982).

${ }^{32}$ T. Biró, H. W. Barz, B. Lukács, and J. Zimányi, Central Research Institute for Physics Budapest Report KFKI-198190, 1981; Phys. Rev. C 27, 2695 (1983).

${ }^{33}$ H. Stöcker, Lawrence Berkeley Laboratory Report LBL12302, 1981.

${ }^{34}$ H. Stöcker, A. A. Ogloblin, and W. Greiner, Z. Phys. A $\underline{303}$, 259 (1981).

${ }^{35}$ P. J. Siemens and J. O. Rasmussen, Phys. Rev. Lett. $\underline{42}, 880$ (1979).

${ }^{36}$ I. Montvay and J. Zimanyi, Nucl. Phys. A316, 490 (1979); H. W. Barz, B. Lukács, J. Zimányi, Gy. Fái, and B. Jakobsson, Z. Phys. A $\underline{\text { 302, }} 73$ (1981). 
${ }^{37}$ A. A. Amsden, A. S. Goldhaber, F. H. Harlow, and J. R. Nix, Phys. Rev. C 17, 2080 (1978).

${ }^{38}$ L. P. Csernai, I. Lovas, J. A. Maruhn, A. Rosenhauer, J. Zimányi, and W. Greiner, Phys. Rev. C 26, 149 (1982).

${ }^{39}$ L. P. Csernai and H. Stöcker, Phys. Rev. C 25, 3208 (1982).

${ }^{40} \mathrm{G}$. Buchwald, G. Graebner, J. Maruhn, and H. Stöcker, University of Frankfurt Report UFTP-104/1983, 1983, submitted to J. Comput. Phys.

41J. I. Kapusta, Phys. Rev. C 24, 2545 (1981); 21, 1301 (1980).

${ }^{42}$ H. W. Barz, L. P. Csernai, and W. Greiner, Phys. Rev. C $\underline{26}$, 740 (1982).

${ }^{43}$ L. P. Csernai, D. Kisdi, and J. Németh, Acta Phys. Acad. Sci. Hung. 38, 89 (1975).

${ }^{44}$ D. K. Scott, in Proceedings of the International Workshop on Gross Properties of Nuclei and Nuclear Excitations X, Hirschegg, 1982, edited by H. Feldmeier (Technische Hochschule, Darmstadt, 1982); R. K. Tripathi, Phys. Rev. C 25, 1114 (1982).

45P. Marmier and E. Sheldon, Physics of Nuclei and Particles (Academic, New York, 1970), Vol. II, pp. 886, 920, and 935.

46L. P. Csernai and W. Greiner, Phys. Lett. 99B, 85 (1981).

${ }^{47}$ V. D. Toneev (private communication).

${ }^{48} \mathrm{H}$. H. Gutbrod and H. G. Ritter, Gesellschaft für Schwerionenforschung Nachrichten 1-1982, 1982, p. 3.

${ }^{49}$ B. Schürmann and M. Chemtob, Z. Phys. A 294, 371 (1980).

50J. D. Bowman, W. J. Swiatecki, and C. F. Tsang (unpublished); J. Hüfner, K. Schaefer, and B. Schürmann, Phys. Rev. C 12,1888 (1975).

51 J. Blachot, J. Crancon, A. Lleres, A. Gizon, and H. Nifenecker, in Proceedings of the International Workshop on Gross Properties of Nuclei and Nuclear Excitations X, Hirschegg, 1982, edited by H. Feldmeier (Technische Hochschule, Darmstadt, 1982); J. Blachot, J. Crancon, J. Genevey, A. Gizon, and A. Lleres, Z. Phys. A 301, 91 (1981); J. Blachot, J. Crancon, H. Nifenecker, A. Lleres, A. Gizon, and J. Genevey, ibid. 303, 85 (1981).

${ }^{52}$ A. Fleury, H. Delagrange, R. del Moral, J. P. Dufour, F. Hubert, M. B. Mauhourat, and Y. Llabador, Contribution to the $X$ th European Conference on the Physics and Chemistry of Complex Nuclear Reactions, Lillehammer, Norway, 1981 (unpublished).

${ }^{53}$ T. J. M. Symons, V. P. Viyogi, G. D. Westfall, P. Doll, D. E. Greiner, H. Faraggi, P. J. Lindstrom, D. K. Scott, H. J. Crawford, and C. McParland, Phys. Rev. Lett. 42, 40 (1979); G. D. Westfall, T. J. M. Symons, D. E. Greiner, H. H. Heckmann, P. J. Lindstrom, J. Mahoney, A. C. Schotter, D. K. Scott, H. J. Crawford, C. McParland, T. C. Aves, C. K. Gelbke, and J. M. Kidd, ibid. 43, 1859 (1979).

54J. D. Bjorken and S. J. Brodsky, Phys. Rev. D 1, 1416 (1970).

${ }^{55}$ E. Farhi, Phys. Rev. Lett. 39, 1587 (1977).

56J. Kapusta and D. Strottman, Phys. Lett. B106, 33 (1981).

57J. Cugnon, J. Knoll, C. Riedel, and Y. Yariv, Phys. Lett. B109, 167 (1982).

${ }^{58}$ M. Gyulassy, K. A. Frankel, and H. Stöcker, Phys. Lett. 110B, 185 (1982).

${ }^{59} \mathrm{H}$. Ströbele et al., Gesellschaft für Schwerionenforschung Report GSI-82-32, 1982; J. W. Harris, R. Brockmann, R. E. Renfordt, F. Riess, A. Sandoval, H. Ströbele, R. Stock, J. Miller, H. G. Pugh, M. Raff, L. S. Schroeder, and K. L. Wolf, Bull. Am. Phys. Soc. 26, 1112 (1981).

${ }^{60} \mathrm{H}$. Stöcker et al., Michigan State University Report MSUCL$409,1983$.

${ }^{61} \mathrm{~W}$. Greiner, in Proceedings of the 3rd Adriatic Europhysics Study Conference on the Dynamics of the Heavy Ion Collisions, Hvar, Yugoslavia, 1981, edited by N. Cindro, R. A. Ricci, and W. Greiner (North-Holland, Amsterdam, 1981), p. 359.

${ }^{62}$ M. Gyulassy and W. Greiner, Ann. Phys. (N.Y.) 109, 485 (1977). 